\title{
Clinical management of SARS patients in ICU
}

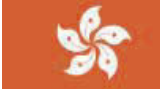

David Chan, RN, MN (Acute Care), ACLS Instructor (AHA), Nurse Specialist (ICU), Prince of Wales Hospital,Hong Kong

e-mail: davidchan123@hotmail.com

Key words: atypical pneumonia critical care nursing severe acute respiratory syndrome (SARS)

\begin{abstract}
In March 2003 Hong Kong was struck by a new atypical pneumonia now known as Severe Acute Respiratory Syndrome or SARS. Many people including citizens and health care workers contracted this disease, and many of them required intensive care unit (ICU) admissions for close observation and management. This article shares the experience of nurses who cared for SARS patients and health workers in the ICU of Prince of Wales Hospital, Hong Kong's largest tertiary referral hospital.
\end{abstract}

\section{INTRODUCTION}

Severe Acute Respiratory Syndrome (SARS) refers to a new type of atypical pneumonia, which was identified and defined by the World Health Organization (WHO, 2003) and the Centre for the Disease Control and Prevention (CDC, 2003) after its first outbreak in Guangdong Province (China) in November 2002.

The outbreak of SARS in Hong Kong could be traced back to Febuary 2003 when a medical doctor (who had a history of treating SARS patients in Guangdong Province, China) came to Hong Kong to attend a wedding ceremony. He stayed in a hotel where he inadvertently spread the SARS virus to many hotel residents. The SARS virus was then spread to the community.

A young man who had stayed at the same hotel was admitted to a medical ward in Prince of Wales Hospital (Hong Kong) in early March with severe flu-like symptoms, which was later diagnosed as SARS. This young man unwittingly spread the SARS virus very quickly to the ward environment via the nebuliser therapy he was receiving, and many health care workers and patients contracted the disease. It soon became clear that a rapidly contagious disease was spreading through this ward and other parts of the hospital. In response the hospital established an 'atypical pneumonia team' to manage the problem.

At about the same time the ICU started admitting patients with atypical pneumonia on March 12-13, but the situation at that time was not identified as an outbreak. On March 14, the ICU received a message from the hospital that there was a large number of patients with atypical pneumonia in the medical wards. The condition of these patients became worse, and many of them required admission to the ICU for closer monitoring and further treatment.

The situation soon became chaotic. The unit needed to arrange for all of the existing patients to be transferred to other hospitals immediately, and at the same time needed to prepare beds for admitting more atypical pneumonia patients with unknown cause. The hospital started mobilizing manpower and resources to the $I C U$, and the ICU itself also started setting up infection control facilities and guidelines to deal with the large number of atypical pneumonia admissions. The ICU was then cohorted as a SARS ICU.

The criteria of ICU admission was those patients having a $\mathrm{SpO}_{2}$ $<90 \%$ (despite a $\mathrm{FiO}_{2}>0.5$ ) and respiratory rate $>35 \mathrm{bpm}$. Most of the patients requiring admission to ICU presented with dyspnoea, hypoxia, tachycardia, mildly deranged liver function and diarrhoea.

From March to June 2003, more than 1700 people have contracted the SARS virus throughout the Hong Kong territories, and up to $20 \%$ of these people required admission to ICU for management due to development of respiratory failure [Chinese University of Hong Kong (CUHK) 2003].

\section{SEVERE ACUTE RESPIRATORY SYNDROME}

Pneumonia can be classified as typical and atypical pneumonia. The usual pneumonia or typical pneumonia is caused by bacteria such as streptococcus. Symptoms include fever, cough, purulent sputum and chest pain. Whereas, atypical pneumonia is usually caused by chlamydia, mycoplasma, legionella, influenza virus, adenovirus and other unknown micro-organisms. Common symptoms include fever, dry cough, chills, headache and general malaise.

SARS is a new kind of atypical pneumonia, and it is caused by coronavirus (Tsang, et al., 2003). A recent study showed that the coronavirus causing SARS in humans may have come from civet cats or some other wild animals (CUHK, 2003).

An early study of 138 SARS patients admitted to Prince of Wales Hospital (PWH) identified a range of common symptoms including: fever (100\%), chills (73\%) and some forms of acute respiratory syndrome, such as myalgia (61\%), dry cough $(57 \%)$, headache $(56 \%)$, dizziness $(43 \%)$ and others (dyspnoea, malaise and diarrhoea) (Lee et al., 2003). SARS can be transmitted by respiratory droplets over a short distance of one metre, or by contact with a patient's respiratory secretion. The incubation period is normally 
5-7 days (but may be up to 10 days). Lab-oratory tests in this same study showed leukopenia (33.9\%), lymphopenia $(69.6 \%)$, thrombocytopenia $(44.8 \%)$, prolonged APTT $(42.8 \%)$, elevated $d-$ dimer (45\%), elevated ALT (23.4\%), elevated CK (32.1\%), elevated LDH $(71 \%)$, hypokalaemia $(25.2 \%)$ and hyponatraemia $(20.3 \%)$. Radiographic findings showed air-space consolidation (78.3) in chest $X$ ray $(\mathrm{CXR})$, or peripheral ground-glass opacification in $\mathrm{CT}$ thorax. (Lee et al., 2003)

Diagnostic tests for SARS (HA, 2003) include serum test for coronavirus antibody titre; and excretions (including tracheal aspirate, tracheal swab, nasopharyngeal aspirate, nasal swab, urine or stool) for reverse transcriptase - polymerase chain reaction (RT-PCR) molecular test. In these SARS patients, the antibody titre showed 40 or above; and the RT-PCR showed a positive result.

Other than providing adequate cardio-respiratory support, the mainstay of treatment included ribavirin and steroids (including hydrocortisone and methylprednisolone). For those patients who were not responding to treatment well, immuno-globulin $M$, convalescence anti-serum, plasma exchange or anti-HIV therapy (e.g. kaletra) were tried.

\section{CARING FOR SARS PATIENTS IN ICU}

It was noted that SARS primarily affected patients' lungs and respiratory function, whereas the effects on other body systems were minimal. Most patients requiring admission to ICU pre-sented with hypoxaemia, tachycardia, mildly deranged liver func-tion and diarrhoea. The following paragraphs describe nursing care of SARS patients in this ICU during the early period of SARS outbreak:

\section{Maintain adequate oxygenation}

Since compromised respiratory function was the most significant problem the primary goal of nursing was to maintain an optimal cardio-respiratory function in order to achieve an adequate oxygenation. Oxygen devices with low gas flow were initially used on SARS patients (e.g. nasal cannula, and Hudson mask). Oxygen devices with high gas flow (e.g. non-rebreathing mask, venturi mask) were not used in this case because their high gas flow might enhance the spread of the SARS virus. The use of non-invasive positive pressure ventilators (NIPPV) (e.g. BiPAP machines) was also prohibited, because BiPAP systems were not closed systems and might also spread patients' expired gas and virus to the environment. If it was a must to use BiPAP systems, patients had to be placed in isolation rooms with negative pressure ventilation, so as to prevent spread of the SARS virus. All staff working inside the isolation room should have the effective personal protection equipment (PPE) (e.g. air mate) before working in such areas. Nebulizers were also avoided where possible to reduce the spread of SARS virus.

Despite the use of oxygen masks, patients could develop oxygen desaturation very rapidly and easily especially when they performed some mild movement or exercise in bed (e.g. turning in bed, CXRs, passing urine or opening bowels in bed). This was managed by temporarily increasing the oxygen concentration until patients resumed their oxygen saturation and planning movements judiciously and only as necessary.

In order to detect any occurrence of respiratory distress, regular arterial blood gas analysis was required. Patients with dyspnoea, cyanosis and oxygen desaturation required endotracheal intubation and mechanical ventilation. Many health care professionals contracted SARS through this procedure. Before proceeding to endotracheal intubation, whole team would put on goggles and a

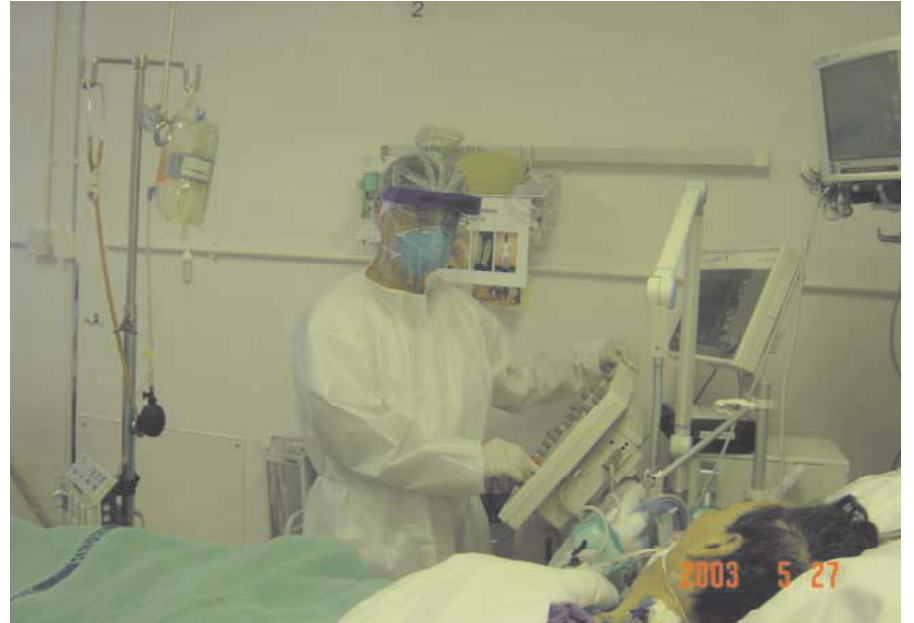

Figure 1. A nurse, who is on standard PPE, is taking care of a ventilated patient.

hood (head cover) on top of the standard PPE. A high efficiency bacterial-viral filter was added to the manual resuscitator to filter patients' expired air during such procedures. To minimize the spread of patients' expired air and virus to the environment and to all the staff, nurses used disposable ventilator tubing, and connected the closed suctioning system, bacterial-viral filter and scavenging evacuation system to the ventilator. Although most SARS patients only had minimal endotracheal secretion, nurses still needed to ensure patency of the patients' airway. Nurses performed endotracheal suctioning regularly and whenever if necessary. The 'closed suctioning system' was used to prevent patients' sputum and virus from spreading. If needed, tracheal aspirate was collected for RT-PCR and culture. Ventilator tubing, in this case, would not be changed until the patient was weaned from the ventilator. To prevent further lung damage, pressure controlled ventilatory modes (e.g. PCV, PRVC) were usually used in these cases. Closed suctioning system and bacterial-viral filter were changed every 48 hours. Heated water humidifiers were not used in this case, because the water bath would enhance the growth of micro-organisms. Instead, heat moisture exchangers were used. To prevent the endotracheal tube (ETT) blocking, it was flushed with normal saline if needed. Nebulization was given via metered-dose inhaler in these ventilated patients.

Since the heated water humidifiers were not allowed to be used, the patient's airway was very dry, and blocked ETT sometimes occurred. Also, nurses needed to avoid unnecessary breaking or disconnecting the breathing circuit, because again this would spread the SARS virus to the environment. If it was really necessary to disconnect the ventilator circuit temporarily (e.g. to add a sputum collection trapper), nurses turned the ventilator to standby mode temporarily or just pressed the ventilator's 'expiratory pause hold' function while disconnecting the tubing, so that the ventilator would not blow out gases during the circuit disconnection. Many SARS patients' respiratory function deteriorated very rapidly even after being put on ventilators. Many ventilated patients developed Acute Respiratory Distress Symptom (ARDS). ARDS has a high mortality, up to $60 \%$ (Brandstetter, et al 1997). Prone ventilation technique was used to improve patients' oxygenation by improving the ventilation-perfusion matching (VQ matching), and to re-expand consolidated lungs. Previous studies have shown that $70 \%$ of patients having prone ventilation had their oxygenation improved (Gattinoni et al, 2001). However, care should be taken to avoid complications that might occur during the prone procedure, such as: displacement of ETT, displacement of intravenous 


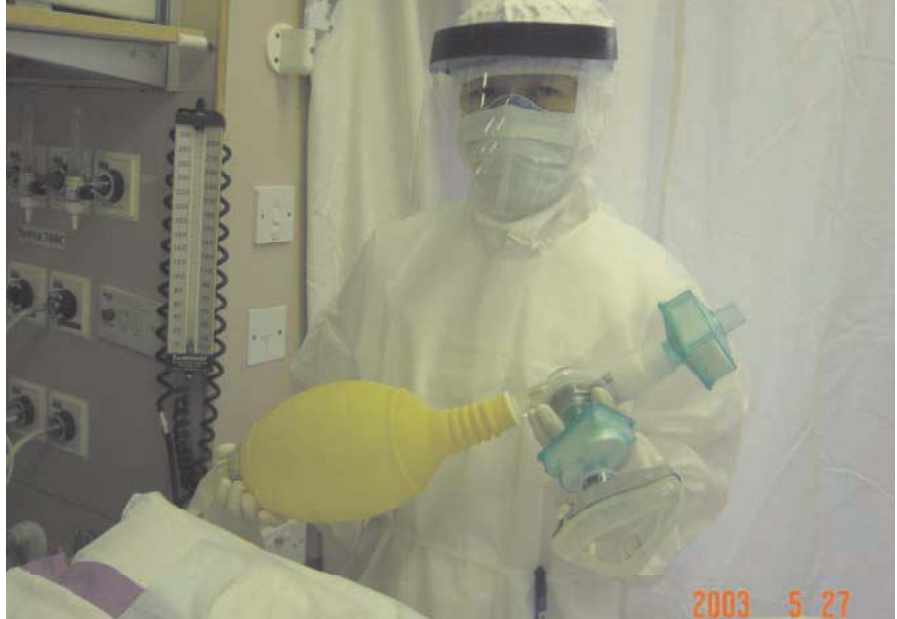

Figure 2. A nurse, who is on standard PPE with a hood, is preparing to ventilate thepatient with a manual resuscitator.

lines, spraining or dislocation injury of the neck and shoulder joints (Chan, 2003). Therefore, adequate planning and preparation before proning the patient are needed.

Our experience has shown that most cases in this incident had their oxygenation improved after proning. No study had been done before to examine what should be the optimal duration for putting a patient in the prone position. The duration could last from a few hours to 1-2 days, depending on the condition and oxygenation status of the individual patient. In our centre, most patients needed to be put in the prone position for 24-48 hours before resuming the supine position for re-assessment.

Weaning was commenced with those patients whose pneumonia resolved, fever came down, and who could breathe spontaneously with a adequate gas exchange.

Generally speaking, SARS itself did not affect patients' cardiovascular function very significantly. Few patients required inotropic support. ribavirin did cause some cardiovascular side effects, such as hypotension, and arrhythmias. In this group of patients having ribavirin, bradycardia with a pulse rate below 60 bpm was common.

Also, nurses observed patients very closely for any development of haemolytic anaemia due to the use of ribavirin. Should haemolytic anaemia occur, patients' haemoglobin and haptoglobin would drop, whereas the reticulocyte count (immature red blood cell) would increase. Moreover, SARS itself would cause a decrease in blood platelets, which could induce a bleeding tendency. Should the above conditions occur, replacement of blood or blood component might be needed.

\section{Monitor neurological function}

Generally speaking, SARS itself did not directly affect patients' neurological function, but many ventilated patients did require sedation and analgesia to keep them calm and comfortable. Nurses needed to check patients' conscious level and degree of comfort regularly to make sure that the dose of sedation was appropriate.

\section{Maintain normal fluid and electrolytes}

In order to maintain a normal fluid balance, nurses recorded the intake (e.g. intravenous fluid intake) and output (e.g. urine output) fluid balance hourly to make sure that any fluid excess or fluid deficit could be detected early and managed properly.

High dose steroid induced hypokalaemia, hypernatraemia and hyperglycemia were common and needed to be monitored and corrected.

\section{Maintain adequate nutrition and normal excretion}

If patients could eat orally, they were allowed to do so, otherwise nasogastric feeding was instituted. Nurses needed to observe patients for any diarrhoea after feeding. Nurses also took special precautions when handling patients' excreta to prevent contraction of the SARS infection. The health care assistants were instructed to dispose the excreta properly to prevent contracting and spreading the SARS virus. To control the diarrhoea, anti-diarrhoea medication (e.g. immodium) was administered.

\section{Ensure adequate protection on skin integrity \& immune} function

a) Skin protection: Because of the poor exercise tolerance, most SARS patients were bed-ridden. Nurses needed to observe patients' skin condition to detect any early onset of possible pressure sore formation. If patients required prone ventilation, the incidence of developing pressure sore would largely be increased, because patients' anterior body part would be very difficult to assess.

b) Immune protection: One first line treatment for SARS was to administer high dose steroids to suppress the patients' immune system, so that the immune system would not attack the host cells. But, problems occurred in many cases, because superimposed nosocomial infection developed after suppression of the immune system. Other than carrying out prescribed treatment, nurses took extra care to protect patients from unnecessary pathogen exposure.

\section{Monitor the physical, psychological and general well being}

It was noted that the activity tolerance of SARS patients was very low. Since high dose steroid induced myopathy (muscle weakness), some patients were very weak in muscle power. Nurses needed to observe patients' muscle power, and offered necessary help (such as help feeding the patient when needed). Passive and active exercises may also be helpful provided they do not tire the patient excessively.

To prevent the development of deep vein thrombosis in bedridden patients, anti-coagulants (e.g. low molecular weight heparin) and anti-embolic stockings were instituted.

A few patients developed an acute confusional state, which could be due to ICU psychosis (Granberg, 1996) or steroid psychosis (Hall, 2003). ICU psychosis could be caused by the following factors: strange and stressful environment, prolonged isolation, sensory overload, sensory deprivation, impaired communication, and altered sleeping pattern. High dose steroids could be due to the disturbance of the sodium/potassium pump of the frontal lobe. In this SARS incidence, family visiting was not allowed to prevent unnecessary spreading of the disease. Telephones were arranged to allow patients to talk to their families if able.

\section{CONCLUSION}

Since the outbreak of SARS between March and June 2003, there have been more than 1700 people who have contracted the SARS virus, with a crude mortality about 10-20\% (CUHK, 2003).

The full range of nursing skill and care is required by the SARS victim in ICU, and safe and effective care is necessary to protect the patient, family and carer in this challenging situation. It is 
hoped that sharing this experience will help other nurses anticipate how they will manage similar outbreaks in their own ICUs.

\section{ACKNOWLEDGEMENT}

The author would like to thank Associate Professor Ged Williams, Divisional Director of Nursing, Royal Melbourne Hospital, Australia for assistance and support on earlier manuscripts leading to this publication.

\section{REFERENCES}

Brandstetter RD, Sharma KC, DellaBadia M et al. (1997). Acute respiratory distress syndrome: a disorder in need of improved outcome. Heart \& Lung; 26: 3-14.

Chan D. (1999). Critical care nursing series: Acute respiratory distress syndrome and prone ventilation. Hong Kong Nursing Journal; 35: 19-26.

Centers for Disease Control and Prevention (CDC). (2003). Severe acute respiratory syndrome (SARS) updated interim case definition. (Accessed Apr 4, 2003, at $h$ ttp:// www.cdc.gov/ncidod/sars/casedefinition.htm)

Chinese University of Hong Kong (2003). Severe acute respiratory syndrome. (Accessed 12 May 2003 at: http://www.aic.cuhk.edu.hk/web8/sudden_acute_ respiratory_syndrom.htm)

Gattinoni L, Tognoni G, Pesenti A et al. (2001). Effect of prone positioning on the survival of patients with Acute Respiratory Failure. The New England Journal of Medicine; 345: 568-573.
Granberg A, Engberg I, Lundberg D. (1996). Intensive Care Syndrome: a literature review. Intensive Care Critical Nursing; 12: 173-182.

Hall RCW. Psychiatric adverse drug reactions: steroid psychosis. (Accessed July 2003, at http://www.drrichardhall.com/steroid.htm

Hospital Authority (HA). (2003). (Accessed at http://www.ha.org.hk/sars/)

Lee N, Hui D, Wu A et al. (2003). A major outbreak of severe acute respiratory syndrome in Hong Kong. (Accesses Apr 7, 2003, at http://www.nejm.org. The New England Journal of Medicine, Apr 7, 2003.)

Pappert D, Rossaint R, Slama K et al. (1994). Influence of positioning on ventilationperfusion relationships in severe acute respiratory distress syndrome. Chest;106: 1511-1516.

Tsang KW, Ho PL, Ooi GC et al. (2003). A cluster of cases of Severe Acute Respiratory Syndrome in Hong Kong. The New England Journal of Medicine. (Accessed Mar 31, 2003, at http://www.nejm.org)

University of Hong Kong (HKU) - Faculty of Medicine. (2003). Breakthrough in the research of SARS associated coronavirus (Accessed May 23, 2003 at http:// www.hku.hk/facmed/press/)

World Health Organization (WHO). (2003). Cumulative number of reported cases (SARS) from 1 Feb to 27 Mar 2003. (Accessed Apr 4, 2003 at http://www.who.int/csr/sarscountry/2003_03_27)

Readers may be interested to know of a very useful reference text (3rd edition) that can be downloaded from the internet free of charge at: http://www.SARSreference.com

What is really useful about this is that there are also a number of translated editions

That can also be downloaded free:

Chinese (1st Edition)

http://www.cmu.edu.cn/research/publications/index.

htm

http://www.chinalabnet.com/SARS/

French (2nd Edition)

http://www.inist.fr/SARS/SARS.php

http://www.inist.fr/SARS/SRAS_F_2ed2.pdf

Italian (2nd Edition)

http://www.sanitaweb.it/web/home/

Romanian (2nd Edition)

http://sarsreference.com/SARS_Reference_2nd_

Romanian.pdf

Spanish (1st Edition)

http://www.iner.gob.mx/ 